\title{
Androgen receptor promotes the migration and invasion of upper urinary tract urothelial carcinoma cells through the upregulation of MMP-9 and COX-2
}

\author{
CHI-CHENG CHEN ${ }^{2 *}$, TENG-FU HSIEH ${ }^{2 *}$, CHAO-HSIANG CHANG $^{1}$, WEN-LUNG MA ${ }^{1}$, \\ XIAO-FAN HUNG ${ }^{1}$, YI-RU TSAI ${ }^{1}$, MENG-HSUEH AMANDA LIN ${ }^{1}$, CAIXIA ZHANG $^{3}$, \\ CHAWNSHANG CHANG ${ }^{1,3}$ and CHIH-RONG SHYR ${ }^{1,4}$
}

\begin{abstract}
${ }^{1}$ Sex Hormone Research Center, China Medical University/Hospital, Taichung 404; ${ }^{2}$ Division of Urology, Department of Surgery, Buddhist Tzu Chi General Hospital, Taichung Branch, Taichung 427, Taiwan, R.O.C.; ${ }^{3}$ George Whipple Lab for Cancer Research, Departments of Pathology, Urology and Radiation Oncology, and The Wilmot Cancer Center, University of Rochester Medical Center, Rochester, NY 14642, USA; ${ }^{4}$ Department of Medical Laboratory Science and Biotechnology, China Medical University, Taichung 404, Taiwan, R.O.C.
\end{abstract}

Received January 29, 2013; Accepted March 29, 2013

DOI: $10.3892 / o r .2013 .2506$

\begin{abstract}
Dysregulated androgen receptor (AR) signaling is implicated in several types of tumor, including carcinomas of the prostate, breast, liver and bladder. However, the contribution of AR to the progression of upper urinary tract urothelial carcinomas (UUTUC) has not been fully investigated. In the present study, we demonstrated that the AR is involved in the metastasis and invasiveness of UUTUC cells. We investigated the role of the AR in UUTUC by using UUTUC-derived BFTC 909 cells. The overexpression of AR promotes the migration and invasion of BFTC 909 cells. Expression of migration/invasion-related genes was increased in BFTC 909 cells overexpressing AR determined by qPCR and western blot analyses. The results showed that AR-enhanced migration and invasion of UUTUC cells are linked to the upregulation of the matrix-degrading enzyme MMP-9 and cyclooxygenase (COX)-2. Subsequently, the blocking of MMP-9 and COX-2 signaling by inhibitors suppressed AR-enhanced cell migra-
\end{abstract}

Correspondence to: Professor Chih-Rong Shyr, Sex Hormone Research Center, China Medical University/Hospital, Taichung 404, Taiwan, R.O.C.

E-mail: chshyr@hotmail.com

Professor Chawnshang Chang, George Whipple Lab for Cancer Research, Departments of Pathology, Urology and Radiation Oncology, and The Wilmot Cancer Center, University of Rochester Medical Center, Rochester, NY 14642, USA

E-mail: chang@urmc.rochester.edu

${ }^{*}$ Contributed equally

Key words: androgen receptor, upper urinary tract urothelial carcinoma, migration, invasion tion and invasion. The results of the present study provide evidence for the first time of the role of AR in the motility and invasion of UUT cancer cells and support the hypothesis that the AR may play a critical role in the establishment of the invasive phenotype in urothelial neoplasia of UUT. Thus, the AR may also serve as a novel biomarker and potential therapeutic target for UUT cancer.

\section{Introduction}

Upper urinary tract urothelial carcinoma (UUTUC) is an aggressive urologic malignancy associated with high morbidity and mortality although it is relatively rare, accounting for approximately $5-10 \%$ of all urothelial tumors (1). The natural history of upper tract UC is different from UC of the bladder with higher incidence of high-grade deeply invasive disease in the upper urinary tract than in the bladder (2). UUTUCCs that invade the muscle wall generally have a very poor prognosis with 5-year specific survival less than $50 \%$ for pT2/pT3 and less than $10 \%$ for pT4 $(1,3)$. The median survival of pT4 patients is only 7-9 months even with radical nephroureterectomy and chemotherapy $(3,4)$. Therefore, UUTUC presents a serious public health problem and a challenge for clinical physicians and basic scientists to find more effective systemic adjuvant therapy to improve the outcome of UUTUC patients.

UUTUC is a male-dominant disease with a male to female ratio of 2:1 to $2.5: 1(5,6)$. Survival of UUTUC patients was significantly influenced by the male gender, age over 80 years, a two-incision operation, location in both the pelviocaliceal system and the ureter, grade III, and stage T3 and T4 with adjusting for gender and age (7). Gender and stage of UUTUC patients were the only independent prognostic factors predictive of overall survival and female gender was associated with a better survival (7). These studies suggest that gender plays an important role in the development and progression of UUTUC. However, gender differences in malignant diseases, including 
UUTUC, are not fully understood and, therefore, more studies are required to elucidate their pathological mechanisms. Androgens and androgen receptor (AR) have been demonstrated to play an important role in male-dominant cancers, including liver and bladder cancer, by affecting tumor initiation and progression (8-10). Therefore, in the present study, we hypothesized that AR also plays a role in the progression of UUTUC and accounts for the high incidence and poor prognosis of UUTUC in males.

During the progression of tumors from primary sites to metastasis, specific proteins and signals are activated to enable cancer cells to detach from neighboring cells, re-orientate their polarity, invade, migrate, survive and proliferate in foreign microenvironments. These proteins include ECM-degrading enzymes, such as matrix metalloproteinases (MMPs) and cathepsins, which help the degradation of the basement membrane and extracellular matrix (ECM) $(11,12)$. There are also signal molecules capable of inducing stress-fiber assembly and contraction for mobility, such as small G-protein Rho and its important downstream effector, the Rho-associated serine/threonine kinase (ROCK), which are also involved in tumor cell migration and invasion (13-16). In UUTUC, the mRNA levels of RhoA and RhoA protein were higher in tumors and metastatic lymph node tissues than in non-tumor tissues, suggesting that RhoA-ROCK 1 signaling is involved in the invasion and metastasis of UUTUC (17). The expression of MMP-2, MMP-9 and TIMP-1 was an independent predictor of high pT stage (18) and elevated expression levels of MMP-9 and MMP-2 were associated with poor prognosis (19).

AR has been shown to regulate MMP-2 and MMP-9 in the prostate (20) and in prostate cancer (21) and AR action in migration is mediated by RhoA-ROCK signaling axis that controls cell motility in prostate cancer (22). The upregulation of cyclooxygenase 2 (COX-2) expression occurs frequently in a variety of different tumors and COX-2, which is also shown to be an important signaling molecule that regulates cell motility $(23,24)$. The AR agonist, dihydrotestosterone (DHT), was shown to increase levels of the vascular inflammatory mediator COX-2 (25).

However, how AR affects the above signals related to the progress of UUTUC remains unclear. In the present study, we investigated this issue by adding $\mathrm{AR}$ overexpression in UUTUC cells to observe the role of AR in cell migration and invasion as well as to examine possible genes involved in the regulatory role of AR in the migration and invasion in UUTUCs.

\section{Materials and methods}

Cell lines and chemicals. The UUTUC cell line BFTC 909 cells (from a UUTUC of a renal pelvis patient) were a generous gift from Dr C.C. Tzeng (26) and were cultured in Dulbecco's modified Eagle's medium, containing 10\% heat-inactivated fetal bovine serum (FBS) at $37^{\circ} \mathrm{C}$ in an atmosphere of $5 \% \mathrm{CO}_{2}$. SV-HUC cells (ureter cells immortalized by SV40, from an 11-year-old male accident victim) were obtained from ATCC and were cultured in Ham's F-12 medium, containing $10 \%$ heat-inactivated $\mathrm{FBS}$ at $37^{\circ} \mathrm{C}$ in an atmosphere of $5 \% \mathrm{CO}_{2}$. To exogenously express AR in cells, a recombinant lentiviral vector containing wild-type AR (pWPI hAR) (27) and a control lentiviral vector expressing the enhanced green fluorescent protein (pWPI) were used to overexpress AR. Lentiviral PWPI-AR/PWPI-control with pMD2.G packaging and psPAX2 envelope plasmids (lentivirus:packaging:envelopeZ 2:1:1) were co-transfected into $293 \mathrm{~T}$ cells. After $48 \mathrm{~h}$ of transfection, target cells were cultured in the presence of viral supernatant containing $8 \mathrm{mg} / \mathrm{ml}$ polybrene (Millipore, Billerica, MA, USA) for $6 \mathrm{~h}$.

MMP-9 inhibitor I was purchased from Calbiochem (Frankfurt, Germany) and celecoxib and casodex were purchased from Sigma-Aldrich (Buchs SG, Switzerland).

Wound-healing migration assay. Cells were seeded onto $35-\mathrm{mm}$ plates until confluence. The plates were scratched using a sterile pipette tip to generate a wound through the confluent monolayer. Cells were analyzed and photographed with a microscope. Images of the cell wound were captured at 0 and $24 \mathrm{~h}$ of migration. The relative migration was calculated by setting the percentage of wound closure in control cells after $24 \mathrm{~h}$ as 1 .

Transwell migration assay. Cells were first harvested from the culture dish and 1.0x10 $10^{4}$ cells in $200 \mu \mathrm{l}$ of serum-free medium were transferred to the Transwell inserts (the top compartment, $8-\mu \mathrm{m}$ pore size) and $750 \mu \mathrm{l}$ of medium was placed in the lower chamber. Following incubation at $37^{\circ} \mathrm{C}$ for $4 \mathrm{~h}$ in a cell culture incubator, cells on the upper surface of the filters were removed with cotton swabs, filters were washed, fixed, and stained with crystal violet. Cells that had moved to the lower surface of the filter were counted under the microscope. Migrated cells in each field were quantified. Results are presented as relative migration by setting the migrating cell number of control cells as 1 .

Transwell invasion assay. Cell invasion through a three-dimensional ECM was assessed by a Matrigel invasion assay using BD Matrigel coated-Transwell with $8.0-\mu \mathrm{m}$ filter membranes. Cells resuspended in $200 \mu 1$ of serum-free medium were plated onto each filter, and $750 \mu$ l of DMEM containing $10 \%$ FBS were added into the lower compartment of the invasion chambers. After $24 \mathrm{~h}$, cells on the upper surface of the filters were removed with cotton swabs, filters were washed in $4 \%$ paraformaldehyde and stained with $1 \%$ crystal violet. Cells that had invaded to the lower surface of the filter were counted under the microscope.

In vitro adhesion to fibronectin assay. Twenty-four-well culture dishes were pre-coated with $80 \mu \mathrm{l}$ of fibronectin $(2.5 \mathrm{mg} / \mathrm{ml})$ adhesion buffer $(0.25 \%$ bovine serum albumin in Hank's balanced salt solution; HBSS) for $30 \mathrm{~min}$ at $37^{\circ} \mathrm{C}$. Cancer cells $\left(1 \times 10^{5}\right)$ were added to each well. After $30 \mathrm{~min}$ at $37^{\circ} \mathrm{C}$ in a $\mathrm{CO}_{2}$ incubator, non-adherent cells were removed by gentle wash with HBSS. Then, the cells were fixed with $2 \%$ paraformaldehyde in $1 \mathrm{X}$ PBS and the adhesive cancer cells were stained with $0.05 \%$ crystal solution. The staining intensity was quantitated with a spectrometer.

Quantitative real-time RT-PCR. Total RNA was extracted from cells using TRIzol (Invitrogen, Carlsbad, CA, USA) and used for first-strand cDNA synthesis. The mRNA levels were 
measured using CFX96 ${ }^{\mathrm{TM}}$ Real-Time system (Bio-Rad Laboratories) using KAPA SYBR ${ }^{\circledR}$ Fast qPCR kits (Kapa Biosystems, Inc., Woburn, MA, USA). Specific primers for MMP-2, MMP-9, RhoA, Rock1, COX-2 and $\beta$-actin were: MMP-2, F: 5'-CCCCAGACAGGTGATCTTGAC-3' and R: 5'-GCTTGCGAGGGAAGAAGTTG-3'; MMP-9, F: 5'-CGC TGGGCTTAGATCATTCC-3' and R: 5'-AGGTTGGATAC ATCACTGCATTAGG-3'; RhoA, F: 5'-TCAAGCCGGAGGT CAACAAC-3' and R: 5'-ACGAGCTGCCCATAGCAGAA-3'; Rock1, F: 5'-ATGAGTTTATTCCTACACTCTACCACT TTC-3' and R: 5'-TAACATGGCATCTTCGACAC TCTAG-3'; COX-2, F: 5'-CCCTTGGGTGTCAAAGGTAA-3' and R: 5'-GCCCTCGCTTATGATCTGTC-3'; and $\beta$-actin, F: 5'-TCA CCCACACTGTGCCCATCTACGA-3' and R: 5'-CAG CGGAACCGCTCATTGCCAATGG-3'. PCR cycling conditions were: $3 \mathrm{~min}$ at $95^{\circ} \mathrm{C}$ for 1 cycle followed by 40 amplification cycles at $95^{\circ} \mathrm{C}$ for $10 \mathrm{sec}$ and $52^{\circ} \mathrm{C}$ (MMP-2, MMP-9, Rock-1, COX-2 and $\beta$-actin), or $62^{\circ} \mathrm{C}$ (RhoA) for $30 \mathrm{sec}$. Expression levels were normalized to $\beta$-actin mRNA level determined by the $2^{-\Delta \Delta \mathrm{CT}}$ method.

Western blot analysis. Cell lysates were resolved by sodium dodecyl sulphate-polyacrylamide gel electrophoresis (SDS-PAGE), transferred to a nitrocellulose membrane and incubated with specific primary antibodies. Protein bands were visualized using horseradish peroxidase (HRP)-conjugated secondary antibodies and enhanced chemiluminescence reagent (Millipore, Bedford, MA, USA) with the Bio-Rad imaging system.

Statistical analysis. Experiments were repeated at least three independent times. Results are expressed as the means \pm SD. Two-tailed unpaired t-test was used to compare the results between the two groups. A P-value of $<0.05$ was considered to indicate a statistically significant difference.

\section{Results}

Addition of AR in BFTC 909 and SV-HUC cells increases cell migration. To determine the role of $\mathrm{AR}$ in the migration of UUTUC cells, we used different UUT urothelial cells, including established UUTUC cells, BFTC 909, and transformed UUTUC cells, SV-HUC. Since these cells express a very low amount of AR, we exogenously expressed AR with viral infection into these cells to examine how AR affects cell migration. As shown in Fig. 1A, contrast-phase images show that the wound area was significantly reduced in BFTC 909-hAR cells with the addition of AR compared to BFTC 909-pWPI cells. Transwell migration assay also showed that SV-HUC-hAR cells migrated more than SV-HUC-pWPI cells (Fig. 1B). These results indicate that AR stimulates UUTUC cell migration either in cancer cells or transformed UC cells.

Addition of AR in BFTC 909 cells increases cell migration. The metastatic process involves several critical steps such as invasion and adhesion (28). We further determined the ability of AR to enhance invasion of UUTUC cells. Consistent with findings in Fig. 1, the addition of AR in BFTC 909-hAR cells increased the number of cells invading through Matrigel-

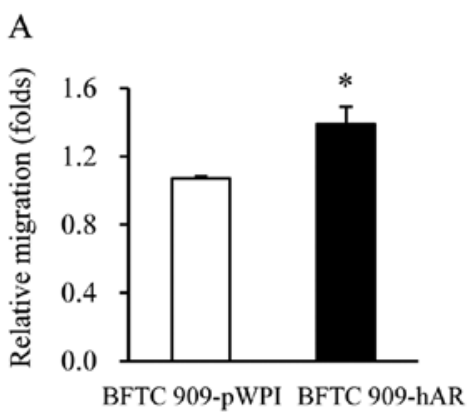

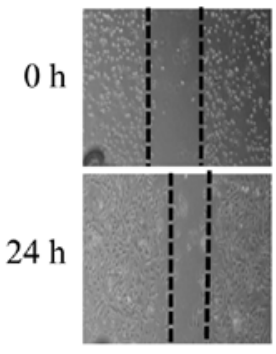

BFTC 909-pWPI

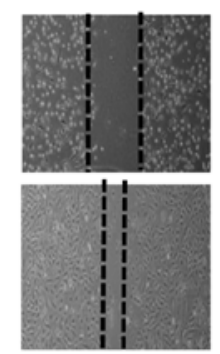

BFTC 909-hAR
B

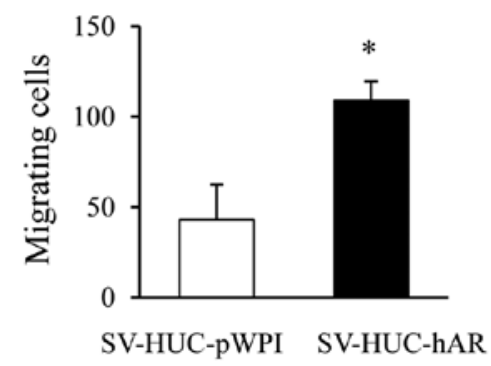

Figure 1. The cell migration assay of UUTUC cells infected with pWPI or hAR by lentiviral system to overexpress AR. For the wound-healing migration assay, cells were plated at high density for $24 \mathrm{~h}$, and then the cell monolayer was scratched and incubated for an additional $24 \mathrm{~h}$ for cell migration into the wound area. Images of the wounds were captured and the recovered areas were calculated. Wound healing was analyzed by comparing the wound healing rate $[\%$ wound healing $=($ Initial wound width - current wound width)/Initial wound width] at 2 different intervals. (A) The quantitative relative migration data and representative microscopic images for BFTC 909-pWPI and BFTC 909-hAR cells. (B) Quantitative relative migration data for SV-HUC-pWPI and SV-HUC-hAR cells by Transwell migration assay. Data represent the means $\pm \mathrm{SD}$. ${ }^{*} \mathrm{P}<0.05$ from at least 3 independent experiments and analyzed by t-test.

coated Transwell filters (Fig. 2A). Adhesion to ECM is an important ability of cancer cells to anchor on the matrix for invasion. Therefore, we also examined the adhesion ability of BFTC 909 cells with or without AR addition. We coated fibronectin, an important component of ECM, on culture plates to investigate the effect of AR on BFTC 909 cell adhesiveness to fibronectin. The addition of AR significantly enhanced the adhesion of BFTC 909-hAR cells compared to BFTC 909-pWPI cells (Fig. 2B). Collectively, our results suggest that AR not only stimulates the invading ability of urothelial cancer cells but also the ability of cells to adhere to cell matrix to promote cell invasion. 
A
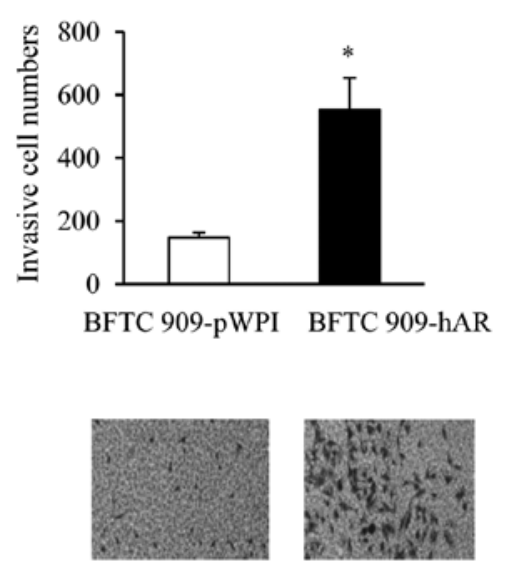

BFTC 909-pWPI BFTC 909-hAR

B

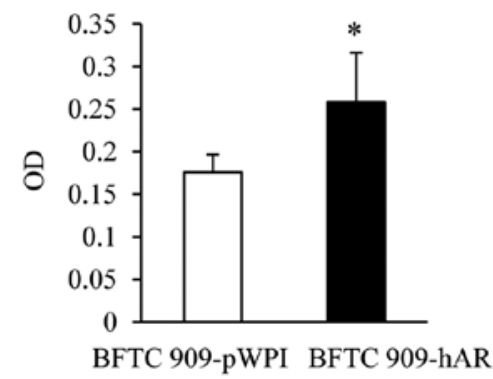

Figure 2. Matrigel invasion and adhesion assay of BFTC 909 cells infected with pWPI or hAR by lentiviral system to overexpress AR. The bottoms of the top well in Transwell setting were all coated with a thin layer of Matrigel and only the cells in the top well with invasive capacity could migrate through the Matrigel layer and $8.0 \mu \mathrm{m}$ pores. The cells with invasion potential through Matrigel-coated Transwells were monitored by crystal violet staining. (A) The quantitative relative migration data and representative microscopic images for BFTC 909-pWPI and BFTC 909-hAR cells. Relative invasion of BFTC 909 hAR cells was normalized to vector-infected cells, set at 1 . (B) For the adhesion assay, BFTC 909-pWPI and BFTC 909-hAR cells were seeded on the culture dishes that were pre-coated with $80 \mu \mathrm{l}$ of fibronectin $(2.5 \mathrm{mg} / \mathrm{ml})$ for $4 \mathrm{~h}$ and then washed with PBS. The adhesive cancer cells stained with $0.05 \%$ crystal solution and the staining intensity was quantitated with a spectrometer. Data represent the means \pm SD of at least 3 independent experiments and were analyzed by t-test. ${ }^{*} \mathrm{P}<0.05$ vs. vector control cells.

Genes related to migration and invasion expression changed by $A R$. To investigate which signaling pathways are activated by AR to increase cell migration and invasion, we examined the several genes which are involved in the migration and invasion of tumor cells. Previous studies have shown that AR stimulates MMP-2 expression in human prostate cancer, which is involved in cell migration (29). AR action in migration is mediated by RhoA-ROCK signaling axis that controls cell motility in prostate cancer (22), which regulates the cytoskeleton and cell migration and is frequently overexpressed in tumors (15). COX-2 is another important signal molecule which regulates cell motility, and AR agonist DHT was shown to increase levels of the vascular inflammatory mediator COX-2 (25). Therefore, we assessed the expression of these genes by qRT-PCR and immunoblot analysis in BFTC 909 cells with or without AR addition. In mRNA levels, the expression of MMP-9, RhoA and Rock-1 was increased, while the expression of MMP-2
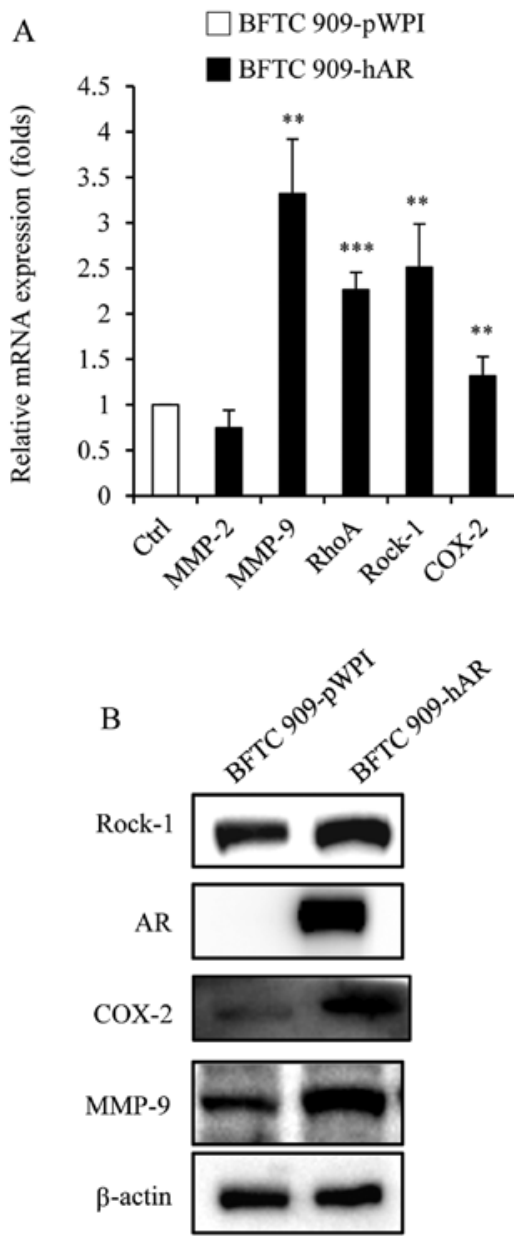

Figure 3. (A) The expression of pro-invasion/metastasis genes on BFTC 909-pWPI and BFTC 909-hAR cells. RNAs from BFTC 909-pWPI and BFTC 909-hAR cells were analyzed by qRT-PCR on mRNA levels of pro-invasion/metastasis genes, MMP-2, MMP-9, Rho, ROCK1 and COX-2. Each transcript level from BFTC 909 pWPI cells was set as 1. Data represent the means \pm SD of 3 independent experiments and were analyzed by t-test. ${ }^{* *} \mathrm{P}<0.01$ and ${ }^{* * *} \mathrm{P}<0.001$ vs. vector-infected control cells. (B) The expression of proteins related to migration and invasion changed by AR expression. BFTC 909-pWPI and BFTC 909-hAR cells. The cells were lysed. Equal amounts of protein were analyzed by SDS-PAGE and immunoblotted with anti-ROCK1, anti-AR, anti-COX-2, anti-MMP-9 and anti- $\beta$-actin antibodies. Representative blots of more than 3 independent studies are shown.

and COX-2 was not changed in BFTC 909-hAR cells, when compared with BFTC 909-pWPI cells (Fig. 3A). Furthermore, in protein levels, BFTC 909-hAR cells had higher expression of MMP-9, Rock-1 and COX-2 than BFTC 909-pWPI cells (Fig. 3B). These results suggest that AR may upregulate these genes at the RNA and protein level to enhance cell migration and invasion.

Inhibitor effects on AR-enhanced cell migration and invasion in UUTUC cells. To further determine the role of MMP-9 and COX-2 in AR-enhanced migration and invasion, we tested whether MMP-9 and COX-2 activities were required for enhancing migration and invasion of BFTC 909-hAR cells by performing the same experiments as above, but in the presence of MMP-9 inhibitor I, a cell-permeable, potent, selective, and reversible MMP-9 inhibitor, or selective COX-2 inhibitor (celecoxib). In the migration assay, as expected, AR-enhanced cell 

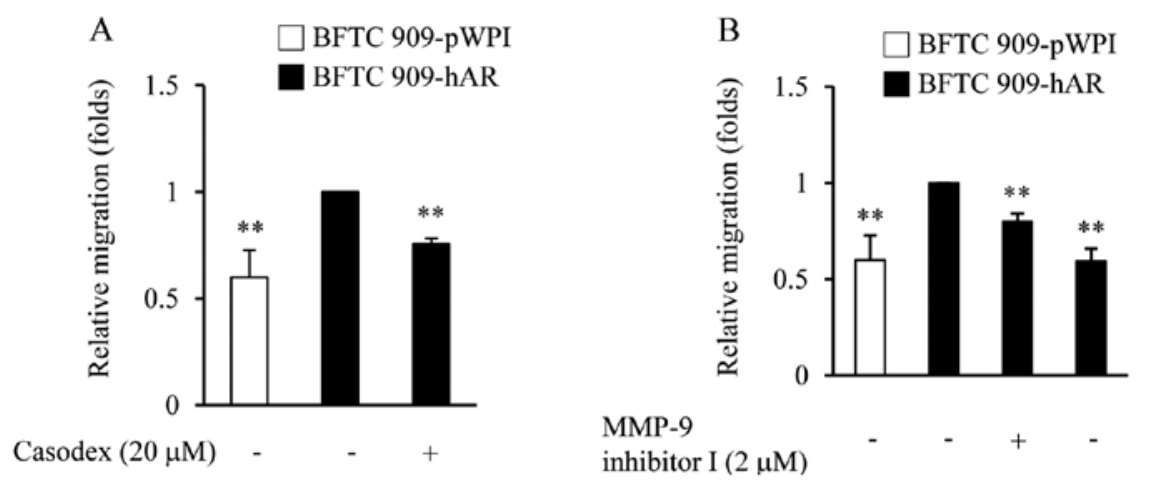

$\begin{array}{lllll}\text { MMP-9 } & - & - & + & - \\ \text { inhibitor I }(2 \mu \mathrm{M}) & - & & & \\ \text { Celecoxib }(80 \mu \mathrm{M}) & - & - & - & +\end{array}$

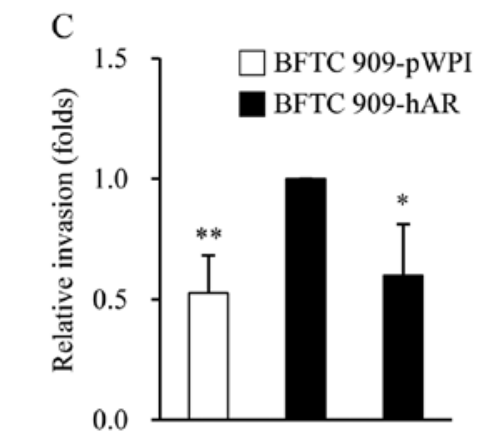

Casodex $(20 \mu \mathrm{M})-\quad-\quad+$

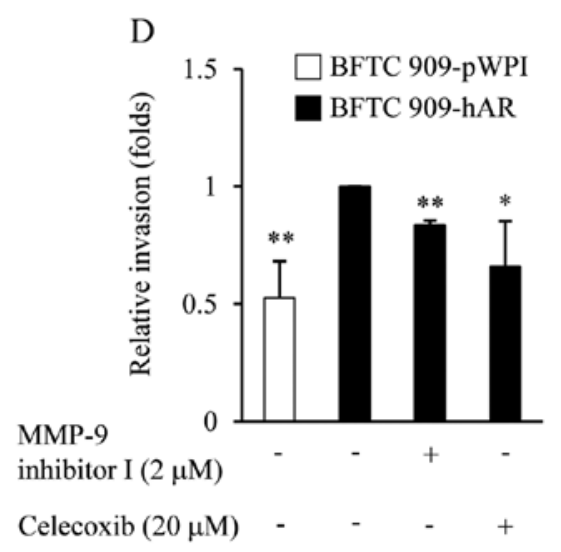

Figure 4. Inhibitor effects on AR-enhanced cell migration and invasion in BFTC 909 cells. The inhibitory effect of (A) casodex and (B) MMP-9-I inhibitor or COX-2 on the AR-enhanced migration of BFTC 909-hAR cells was assessed by wound healing assay. BFTC 909-hAR cells were incubated with casodex, MMP-9-I and COX-2 inhibitors for $24 \mathrm{~h}$. Relative quantification of wound repair of each inhibitor treatment group was quantified by setting the migration rate of no treatment BFTC 909-hAR cell group as 1. The inhibitory effect of (C) casodex, (D) MMP-9-I inhibitor, or COX-2 inhibitor on the AR-enhanced invasion of BFTC 909-hAR cells was assessed by Transwell invasion assay. BFTC 909-hAR cells were incubated with casodex, MMP-9-I or COX-2 inhibitors for $24 \mathrm{~h}$ in Transwell plates coated with a thin layer of Matrigel. Relative quantification of cell invasion of each inhibitor treatment group was quantified by setting invading cell number of no treatment BFTC 909-hAR cell group as 1 . Values are the means \pm SD from at least 3 independent experiments. "P<0.05, ${ }^{* *} \mathrm{P}<0.001$ vs. untreated BFTC $909-\mathrm{hAR}$ cells.

migration in BFTC 909-hAR cells was suppressed by the antiandrogen, casodex (Fig. 4A). Furthermore, MMP-9 and COX inhibitors also showed the ability to suppress AR-enhanced cell migration (Fig. 4B). In the invasion assay, BFTC 909-hAR cells that invaded Matrigel to the lower surface of the filter were decreased by casodex (Fig. 4C) and MMP-9 and COX-2 inhibitors also had the same effect (Fig. 4D). The finding that both migration and invasion were markedly reduced in the presence of casodex, MMP-9 inhibitor and celecoxib, indicate that AR, MMP-9, COX-2 signaling are involved in the ability of AR to promote migration and invasion of BFTC 909 cells.

\section{Discussion}

The present study indicated that AR plays a role in the migration and invasiveness of UUTUC cells, based on the results that there is increased cell migration and invasion following AR addition in UUTUC cells (Figs. 1 and 2). Our findings may aid in clarifying the clinical implications of androgen and $\mathrm{AR}$ signaling in the progression of UUTUC, which may explain the higher male ratio in invasive UUTUC. Although our previous study showed that approximately $40 \%$ of invasive UUTUCs were AR positive (30), the correlation of AR status with relapse and metastasis following radical nephroureterectomy, and survival of patients with invasive UUTUC, has not been investigated. Whether AR is critical in influencing UUTUC progress and survival outcome requires further investigation. In the present study, we provided evidence that AR promotes UUTUC metastasis involving induction of MMP-9 and COX-2 in UUTUC cells, all of which have well established roles in cancer metastasis $(6,11,12)$.

AR expression levels in the tumor and/or its microenvironment affect prostate cancer metastasis $(31,32)$. Exogenous expression of AR in AR-negative PC-3 prostate cancer cells decreased their invasive properties, and treatment with androgen further reduced invasion of these cells (33), but AR functions in prostate stromal cells as a promoter for prostate cancer proliferation and metastasis (34). Although the role of AR in UUTUC has not been investigated, in UC of bladder, AR was shown to promote BBN-induced bladder cancer in mice (9) and bladder cancer cell migration and invasion (35), suggesting AR also plays an important role in urothelial carcinoma. Our study is the first to show the role of AR in increasing UUTUC cell migration and invasion, which is in contrast to the effect of AR on prostate cancer, although others also reported that AR promotes the invasiveness of prostate cancer cells $(21,29)$. These studies indicate that AR may have diverse effects on molecules involved in cancer invasion and 
metastasis by suppressing or stimulating cancer cell migration and invasion, which may be due to the tumor microenvironment, coregulators of AR and alterations of growth factors and their receptors in tumors (36). The exact mechanisms by which AR exerts its effects in different cancer cells requires further investigation in order to dissect the multiple roles of $\mathrm{AR}$ in cancer progression and metastasis.

In delineating the molecules regulated by AR in affecting UUTUC metastasis, we examined the genes including MMPs involved in the degradation of the ECM, which is a key step in the process of cancer invasion and metastasis. Our results showed that among different MMPs, MMP-2 and MMP-9, AR increased MMP-9 expression both at the mRNA and protein level, but not MMP-2 in UUTUC cells (Fig. 3A). In prostate cancer cells, regulation of MMP-2 or MMP-9 by AR signaling has different results from different groups. Some studies have reported that androgen stimulates pro-MMP-2 expression but not pro-MMP-9 in LNCaP cells (29) and both MMP-2 and MMP-9 are stimulated by AR signaling in MDA-I cells (21). However, Miyamoto et al reported that androgen decreases MMP-9 secretion in PC-3 cells stably expressing AR (37). Therefore, the molecular mechanism on the upregulation of MMP-9 expression of AR requires further analysis, but the inhibitors of MMP-9 were shown able to block AR-enhanced cell migration and invasion. Since MMP inhibitors have been proposed as promising targets for cancer therapy (38), the combination of AR antagonists is likely to increase treatment efficacy in AR positive UUTUC cells.

To metastasize, tumor cells need to increase motility by remodelling the cytoskeletons and cell contacts with the ECM, which is regulated by RhoA and ROCK-1 kinase (15). Although we have demonstrated the increase of RhoA and ROCK-1 expression in BFTC 909 cells with addition of AR, the inhibitors of ROCK-1 failed to block AR-enhanced migration and invasion (data not shown), suggesting that RhoA and ROCK-1 may not significantly affect AR function. The higher expression level of COX-2 has been shown to increase invasiveness in colon cancer and prostate cancer cells $(24,39)$. For UUTUC, overexpressed COX-2 was also found in patients and was associated with the pathologic stage and grade, indicating that COX-2 may be involved in UUTUC carcinogenesis and development $(40,41)$. The effect of the specific COX-2 inhibitor on AR-enhanced migration and invasion clearly demonstrated the essential role of COX-2 in cell migration and invasion (Fig. 4B and D).

Inhibition of COX-2 and MMP-9 suppressed AR-enhanced cell migration and invasion in UUTUC (Fig. 4B and D). This finding provides the rationale to develop new therapeutic treatment by combining AR blockade and chemotherapy or target therapy, which may produce better efficacy. Since COX-2 and MMP-9 are also associated with tumor progression, inhibition of the COX-2 and MMP-9 pathways could be an effective therapeutic approach for advanced UUTUCs. The combined therapy of androgen deprivation and other anti-AR therapies with inhibition of the COX-2 and MMP-9 pathways may have improved therapeutic efficacy on advanced UUTUCs. Therefore, our study has several important clinical implications; first, our data proved that the expression of AR is linked to tumor cell migration and invasion, although whether AR overexpression in UUTUCs is associated with higher clinical stage and poor clinical outcome requires further investigation. Second, our study suggested that the addition of AR blockade in therapeutic regimens combined with targeted drugs may have better responses in UUTUC patients who have AR positive tumors. Adjuvant chemotherapy following surgery is currently used in UUTUC patients to prevent cancer relapse and metastasis, but adjuvant chemotherapy only achieves a 5 -year recurrence-free rate of up to $50 \%$ with minimal impact on survival $(42,43)$. Since AR blockade has been commonly used and has been shown to improve survival of men with locally advanced and high-grade prostate cancer (44), the combination of AR blockade with either traditional chemotherapy or target therapy may increase the efficacy of therapeutic regimens on advanced UUTUCs.

\section{Acknowledgements}

This study was supported by the grant (TTCRD-10110) from Buddhist Tzu Chi General Hospital, Taichung, Taiwan. This study was also supported in part by Taiwan Department of Health Clinical Trial and Research Center of Excellence (DOH100-TD-B-111-004).

\section{References}

1. Roupret M, Zigeuner R, Palou J, et al: European guidelines for the diagnosis and management of upper urinary tract urothelial cell carcinomas: 2011 update. Eur Urol 59: 584-594, 2011.

2. Stewart GD, Bariol SV, Grigor KM, Tolley DA and McNeill SA: A comparison of the pathology of transitional cell carcinoma of the bladder and upper urinary tract. BJU Int 95: 791-793, 2005.

3. Li CC, Chang TH, Wu WJ, et al: Significant predictive factors for prognosis of primary upper urinary tract cancer after radical nephroureterectomy in Taiwanese patients. Eur Urol 54: 1127-1134, 2008

4. Hall MC, Womack JS, Roehrborn CG, Carmody T and Sagalowsky AI: Advanced transitional cell carcinoma of the upper urinary tract: patterns of failure, survival and impact of postoperative adjuvant radiotherapy. J Urol 160: 703-706, 1998.

5. Munoz JJ and Ellison LM: Upper tract urothelial neoplasms: incidence and survival during the last 2 decades. J Urol 164: 1523-1525, 2000

6. Ozsahin M, Zouhair A, Villa S, et al: Prognostic factors in urothelial renal pelvis and ureter tumours: a multicentre Rare Cancer Network study. Eur J Cancer 35: 738-743, 1999.

7. Papatsoris AG, Chrisofos M, Skolarikos A, et al: Upper urinary tract transitional cell carcinoma. A 10 -year experience. Tumori 94: 75-78, 2008.

8. Wu MH, Ma WL, Hsu CL, et al: Androgen receptor promotes hepatitis B virus-induced hepatocarcinogenesis through modulation of hepatitis B virus RNA transcription. Sci Transl Med 2: 32ra35, 2010

9. Miyamoto H, Yang Z, Chen YT, et al: Promotion of bladder cancer development and progression by androgen receptor signals. J Natl Cancer Inst 99: 558-568, 2007.

10. Ma WL, Hsu CL, Wu MH, et al: Androgen receptor is a new potential therapeutic target for the treatment of hepatocellular carcinoma. Gastroenterology 135: 947-955, 2008

11. Himelstein BP, Canete-Soler R, Bernhard EJ, Dilks DW and Muschel RJ: Metalloproteinases in tumor progression: the contribution of MMP-9. Invasion Metastasis 14: 246-258, 1994.

12. Kupferman ME, Fini ME, Muller WJ, Weber R, Cheng Y and Muschel RJ: Matrix metalloproteinase 9 promoter activity is induced coincident with invasion during tumor progression. Am J Pathol 157: 1777-1783, 2000.

13. Riento K and Ridley AJ: Rocks: multifunctional kinases in cell behaviour. Nat Rev Mol Cell Biol 4: 446-456, 2003.

14. Schmitz AA, Govek EE, Bottner B and Van Aelst L: Rho GTPases: signaling, migration, and invasion. Exp Cell Res 261: $1-12,2000$.

15. Sahai E and Marshall CJ: Differing modes of tumour cell invasion have distinct requirements for Rho/ROCK signalling and extracellular proteolysis. Nat Cell Biol 5: 711-719, 2003. 
16. Itoh K, Yoshioka K, Akedo $\mathrm{H}$, Uehata M, Ishizaki $\mathrm{T}$ and Narumiya S: An essential part for Rho-associated kinase in the transcellular invasion of tumor cells. Nat Med 5: 221-225, 1999.

17. Kamai T, Kawakami S, Koga F, et al: RhoA is associated with invasion and lymph node metastasis in upper urinary tract cancer. BJU Int 91: 234-238, 2003.

18. Miyata Y, Kanda S, Nomata K, Hayashida Y and Kanetake H: Expression of metalloproteinase-2, metalloproteinase-9, and tissue inhibitor of metalloproteinase-1 in transitional cell carcinoma of upper urinary tract: correlation with tumor stage and survival. Urology 63: 602-608, 2004.

19. Inoue K, Kamada M, Slaton JW, et al: The prognostic value of angiogenesis and metastasis-related genes for progression of transitional cell carcinoma of the renal pelvis and ureter. Clin Cancer Res 8: 1863-1870, 2002.

20. Delella FK, Justulin LA Jr and Felisbino SL: Finasteride treatment alters MMP-2 and -9 gene expression and activity in the rat ventral prostate. Int J Androl 33: e114-e122, 2010.

21. Hara T, Miyazaki H, Lee A, Tran CP and Reiter RE: Androgen receptor and invasion in prostate cancer. Cancer Res 68 1128-1135, 2008

22. Schmidt LJ, Duncan K, Yadav N, et al: RhoA as a mediator of clinically relevant androgen action in prostate cancer cells. Mol Endocrinol 26: 716-735, 2012.

23. Wolff H, Saukkonen K, Anttila S, Karjalainen A, Vainio H and Ristimaki A: Expression of cyclooxygenase-2 in human lung carcinoma. Cancer Res 58: 4997-5001, 1998.

24. Tsujii M, Kawano S and DuBois RN: Cyclooxygenase-2 expression in human colon cancer cells increases metastatic potential Proc Natl Acad Sci USA 94: 3336-3340, 1997.

25. Osterlund KL, Handa RJ and Gonzales RJ: Dihydrotestosterone alters cyclooxygenase-2 levels in human coronary artery smooth muscle cells. Am J Physiol Endocrinol Metab 298: E838-E845, 2010.

26. Tzeng CC, Liu HS, Li C, et al: Characterization of two urothelium cancer cell lines derived from a blackfoot disease endemic area in Taiwan. Anticancer Res 16: 1797-1804, 1996.

27. Ma WL, Hsu CL, Yeh CC, et al: Hepatic androgen receptor suppresses hepatocellular carcinoma metastasis through modulation of cell migration and anoikis. Hepatology 56: 176-185, 2012.

28. Langley RR and Fidler IJ: The seed and soil hypothesis revisited - the role of tumor-stroma interactions in metastasis to different organs. Int J Cancer 128: 2527-2535, 2011.

29. Liao X, Thrasher JB, Pelling J, Holzbeierlein J, Sang QX and Li B: Androgen stimulates matrix metalloproteinase-2 expression in human prostate cancer. Endocrinology 144: 1656-1663, 2003.

30. Shyr CR, Chen CC, Hsieh TF, et al: The expression and actions of androgen receptor in upper urinary tract urothelial carcinoma (UUTUC) tissues and the primary cultured cells. Endocrine 43: 191-199, 2013.
31. Heinlein CA and Chang C: Androgen receptor in prostate cancer. Endocr Rev 25: 276-308, 2004.

32. Niu Y, Altuwaijri S, Lai KP, et al: Androgen receptor is a tumor suppressor and proliferator in prostate cancer. Proc Natl Acad Sci USA 105: 12182-12187, 2008.

33. Bonaccorsi L, Carloni V, Muratori M, et al: Androgen receptor expression in prostate carcinoma cells suppresses alpha6beta4 integrin-mediated invasive phenotype. Endocrinology 141: 3172-3182, 2000

34. Niu Y, Altuwaijri S, Yeh S, et al: Targeting the stromal androgen receptor in primary prostate tumors at earlier stages. Proc Natl Acad Sci USA 105: 12188-12193, 2008.

35. Wu JT, Han BM, Yu SQ, Wang HP and Xia SJ: Androgen receptor is a potential therapeutic target for bladder cancer. Urology 75: 820-827, 2010

36. Baldi E, Bonaccorsi L and Forti G: Androgen receptor: good guy or bad guy in prostate cancer invasion? Endocrinology 144: 1653-1655, 2003.

37. Miyamoto H, Altuwaijri S, Cai Y, Messing EM and Chang C: Inhibition of the Akt, cyclooxygenase-2, and matrix metalloproteinase-9 pathways in combination with androgen deprivation therapy: potential therapeutic approaches for prostate cancer. Mol Carcinog 44: 1-10, 2005.

38. Coussens LM, Fingleton B and Matrisian LM: Matrix metalloproteinase inhibitors and cancer: trials and tribulations. Science 295: 2387-2392, 2002

39. Kirschenbaum A, Liu X-H, Yao S and Levine AC: The role of cyclooxygenase-2 in prostate cancer. Urology 58: 127-131, 2001.

40. Oku S, Higashi M, Imazono Y, et al: Overexpression of cyclooxygenase-2 in high-grade human transitional cell carcinoma of the upper urinary tract. BJU Int 91: 109-114, 2003.

41. Jeon HG, Jeong IG, Bae J, et al: Expression of Ki-67 and COX-2 in patients with upper urinary tract urothelial carcinoma. Urology 76: 513.e7-513.e12, 2010.

42. Vassilakopoulou M, de La Motte Rouge T, Colin P, et al: Outcomes after adjuvant chemotherapy in the treatment of high-risk urothelial carcinoma of the upper urinary tract (UUT-UC): results from a large multicenter collaborative study. Cancer 117: 5500-5508, 2011.

43. Hellenthal NJ, Shariat SF, Margulis V, et al: Adjuvant chemotherapy for high risk upper tract urothelial carcinoma: results from the Upper Tract Urothelial Carcinoma Collaboration. J Urol 182: 900-906, 2009

44. Miyamoto H, Messing EM and Chang C: Androgen deprivation therapy for prostate cancer: current status and future prospects. Prostate 61: 332-353, 2004. 